\title{
Development of Novel Capillary Coating Based on Physical Adsorption for Capillary Electrophoresis
}

\author{
Hiroyuki KaTaYama ${ }^{\dagger}$, Yasushi IshiHama and Naoki AsaKaWA \\ Chemical Analysis Division, Analytical Research Laboratories, Eisai Co. Ltd., \\ Tokodai, Tsukuba, Ibaraki 300-26, Japan
}

Keywords Stable coating, polybrene, dextran sulfate, $\mathrm{pH}$-independent electroosmotic flow

A simple and stable capillary coating based on physical adsorption called "successive multiple ionic-polymer layer (SMIL)" coating was developed and evaluated. ${ }^{1}$ An ionic polymer dextan sulfate (DS) was adsorbed to the inner wall of a capillary by sandwiching cationic polymer polybrene (PB) between the DS and the unmodified capillary wall. The DS coated capillary was expected to show $\mathrm{pH}$-independent electroosmotic flow (EOF) because DS has sulfonic groups. The endurance of the SMIL coating was improved in comparison with the conventional physical adsorption. The $\mathrm{pH}$-independent EOF of the DS coated capillary showed new applications especially in acidic regions where EOF of the uncoated capillary could not be generated.

\section{Experimental}

\section{Materials}

The coating reagent polybrene (PB) was from Fluka (Tokyo, Japan) and dextran sulfate (DS) was from Sigma (St. Louis, MO, USA). Phenol, $p$-toluensulfonic acid, acetylsalicylic acid (aspirin) and quinine hydrochloride were from Wako (Osaka, Japan). Formamide (Pharmacia Biotech, Uppsala, Sweeden) was used as an EOF marker. Others were of reagent grade.

\section{Equipment}

CE was performed with a Beckman P/ACE 2100 (Beckman Instruments, Fullerton, CA, USA). The uncoated fused silica capillaries of $75 \mu \mathrm{m}$ i.d., $27 \mathrm{~cm}$ were from GL Sciences (Tokyo, Japan). Capillaries were thermostated at $25^{\circ} \mathrm{C}$ both during the coating and analyses. Sample injections were performed by pressure $(0.5 \mathrm{psi} ; 1 \mathrm{psi}=6894.76 \mathrm{~Pa})$ for $2.0 \mathrm{~s}$. Coating steps for SMIL were performed by the rinse function of the Beckman CE system.

$\doteqdot$ To whom correspondence showld be addressed.

\section{Procedure of SMIL coating}

Procedure of SMIL coating was performed as follows. The capillaries were rinsed by $1.0 \mathrm{~mol} / \mathrm{l}$ sodium hydroxide, followed by rinsing the aqueous PB solution as the first layer and the aqueous DS solution as the second layer. CE analyses with the SMIL coated capillaries were performed after rinsing by pure water.

\section{Results and Discussion}

When PB solution was flushed through the capillary, EOF was found to be reversed. Then, when DS solution was flushed over PB layer, EOF was reversed again.

A disadvantage of physical adsorption coating is its low endurance ${ }^{2}$, while it has advantages of simple coat-

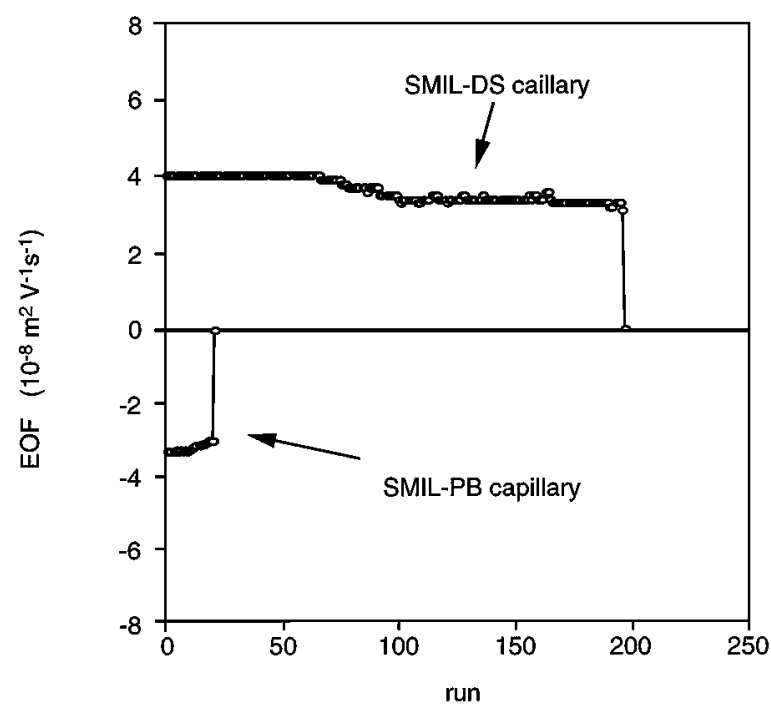

Fig. 1 The endurance of SMIL-PB and SMIL-DS capillary. Conditions: applied voltage, $+7 \mathrm{kV}$; buffer, phosphate buffer at pH $3.0(I=0.05)$; capillary, $75 \mu \mathrm{m}$ i.d. $\times 27 \mathrm{~cm}(20 \mathrm{~cm}$ effective length). 


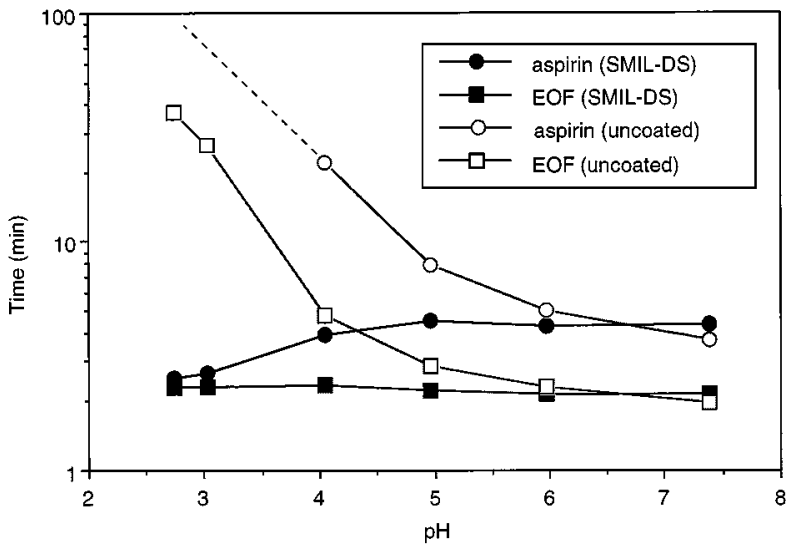

Fig. 2 Dependence of migration time of EOF and aspirin on pH. Conditions: detection, $214 \mathrm{~nm}$; applied voltage, $7 \mathrm{kV}$; buffers, phosphate buffer at $\mathrm{pH} 2-3(I=0.05)$, acetate buffer at $\mathrm{pH} 4-5(I=0.05)$, and phosphate buffer at $\mathrm{pH} 6-7$ $(I=0.05)$; capillary, $75 \mu \mathrm{m}$ i.d. $\times 27 \mathrm{~cm}(20 \mathrm{~cm}$ effective length).

ing procedure and good reproducibility. We overcame this disadvantage by adsorbing the opposite charged polymers successively. The endurance test against the continuous analysis of conventional PB adsorbed capillary (SMIL-PB capillary) and DS adsorbed capillary (SMIL-DS capillary) was performed. The result is shown in Fig. 1. A PB layer of SMIL-PB capillary was detached within 20 runs, while SMIL-DS capillary endured about 200 runs. The remarkable increase of the endurance is probably due to the strong interaction between the PB and the DS layers.

Comparison of EOF and mobility of aspirin between uncoated capillary and SMIL-DS capillary was investigated (Fig. 2). It was quite time-consuming to detect EOF and aspirin $\left(\mathrm{p} K_{\mathrm{a}} 3.48\right)$ below $\mathrm{pH} 4$ when uncoated capillary was used because the silanol groups of the capillary wall were suppressed. On the other hand, those two peaks were detected in a short time when SMIL-DS capillary was used. This is because SMILDS capillary had $\mathrm{pH}$-independent EOF.

The presence of EOF under acidic conditions enabled us to perform further new applications. The separation of cationic, neutral and anionic analytes was performed at $\mathrm{pH}$ 2.75. The result is shown in Fig. 3. It was only possible to detect cationic quinine when uncoated cap-

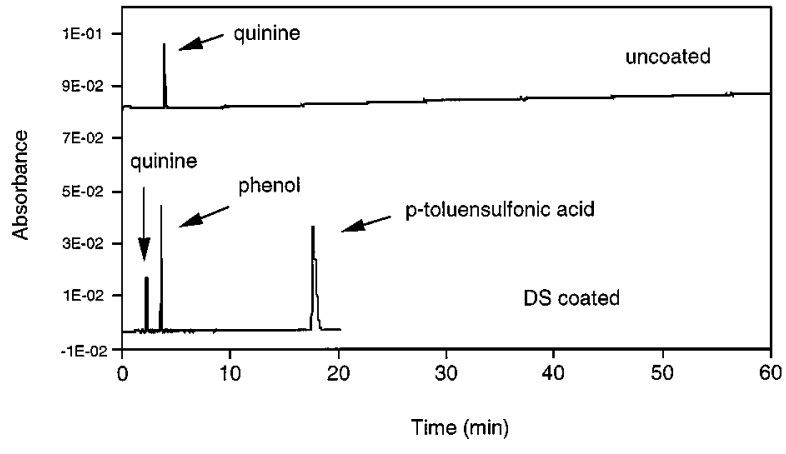

Fig. 3 Separation of the cationic, anionic and neutral compounds under the acidic condition ( $\mathrm{pH}$ 2.75). Conditions: applied voltage, $+7 \mathrm{kV}$; buffer, phosphate buffer at $\mathrm{pH} 2.75$ $(I=0.05)$; capillary, $75 \mu \mathrm{m}$ i.d. $\times 27 \mathrm{~cm}(20 \mathrm{~cm}$ effective length).

illary was used because EOF was suppressed under the acidic condition. However, pH-independent EOF of SMIL-DS capillary enabled to detect cationic quinine, neutral phenol and anionic $p$-toluenesulfonic acid simultaneously. Further new applications were expected by using SMIL-DS capillary.

Such flexibility is one of the great advantages of SMIL coating. It would be possible to achieve stable cationic coated capillary by adsorbing cationic polymer as a third layer. Most of the coated capillary had no EOF because neutral polymer was covalently bonded to the capillary wall. To the contrary, SMIL coated capillary always had EOF. Having EOF from both cathode to anode and anode to cathode would be possible by SMIL coating. We will apply SMIL coated capillary to capillary electrophoresis/mass spectrometry (CE/MS) in the near future where the presence of EOF is quite important to perform the analyses.

\section{References}

1. H. Katayama, Y. Ishihama and N. Asakawa, HPLC 97', Birmingham, UK, Abstract A, p.149, June 1997.

2. E. Cordova, J. Gao and G. M. Whitesides, Anal. Chem., 69 1370 (1997).

(Received December 26, 1997) (Accepted February 2, 1998) 